\title{
Assessment of absorptive capacity
}

\section{By H. Newey and D. H. Smyth, Department of Physiology, University of Sheffield}

The methods for investigating intestinal absorption range from techniques applicable to the human subject to techniques using isolated villi. No one technique provides the answer to all the questions and knowledge must be built up from information obtained by very different techniques. These can be divided into two groups, in vivo and in vitro, the difference being that in the former group the intestine has its own blood supply, while in the latter the intestine or parts of the intestine depend for oxygenation and nutrient materials on the solution in which they are bathed. The in vivo techniques have been classified by Smyth (I96I) according to the extent to which they depart from physiological conditions, and the different principles used. They include such procedures as intubation techniques in the human subject, tolerance curves, absorption from isolated loops of intestine, etc. 'The in vitro techniques date from the work of Fisher \& Parsons (r949), and a further major advance was made by the introduction of the everted sac technique by Wilson \& Wiseman (1954). These in vitro techniques have been discussed by Quastel (I96I), Wilson (rg62) and Smyth (1963).

In the space available it would not be profitable to attempt to describe all the different techniques, and it is more useful to discuss some of the methodological problems still unsolved, one of the most important of which is the assessment of absorptive function in different conditions. This raises the fundamental problem of the best parameter for expressing absorption, a problem which can be looked at from several different points of view. It might be important to know whether more or less of a particular nutrient is absorbed in different conditions, it might be a matter of how to introduce into the body most effectively a particular therapeutic substance, or it might be how cellular energy is effectively utilized for the absorptive process. Although it might not always be relevant, one approach to the problem is consideration of the work done in intestinal transfer. It is convenient to begin with this and see how far it is applicable to the intestine. In the space available it will only be possible to deal with solute transfer, and fluid transfer will only be discussed as it affects solute movement.

\section{Transfer between two compartments}

Consider first the simple case of solutions in two compartments $\mathrm{A}$ and $\mathrm{B}$, with solute being transferred from $A$ to $B$. In this instance the problem could be expressed in thermodynamic terms, and the work done is given by the equation

$$
W=2 \cdot 3 N R T \log \frac{C_{B}}{C_{A}},
$$

where $W$ is the work in calories, $N$ the number of moles transferred, $R$ the gas constant, $T$ the absolute temperature and $C_{A}$ and $C_{B}$ the final activity in compartments $A$ and $B$ respectively. For the sake of simplicity we will equate activities with 
concentrations. There are then three variables to measure, the total amount of solute transferred and two concentrations. The amount of solute can be regarded as a capacity factor, the concentration ratio as an intensity factor. If the substance being transferred is an ion and there is an electrical potential difference between the compartments, then another term $E F$ must be introduced, i.e. the voltage $(E)$ multiplied by the total charge transferred. If we ignore the electrical complication, which will be discussed separately, the assessment of absorptive work by the intestine must be related to equation $\mathrm{I}$. In the intestine, however, it is never possible to apply equation $I$ accurately because the conditions are too complicated, and the main complications are (I) that there are not two compartments only and ( 2 ) there is seldom movement of solute without simultaneous movement of solvent.

\section{Number of compartments}

In absorption, the substance transferred enters the epithelial cells from the intestinal lumen. There is in some part of the cell, we do not know where, a mechanism for concentrating it. If the mechanism is at the 'serosal' pole of the epithelial cell (i.e. at the opposite side to the luminal border) the highest concentration of solute will be produced in the subepithelial space. If the mechanism is at some other part of the cell, the highest concentration is produced in some region of the cell. As a result of this high concentration diffusion takes place from the cell into the subepithelial space, and it must be assumed that there is a greater barrier to diffusion towards the luminal side than towards the serosal side. Again a high concentration of solute is created in the subepithelial fluid in the lamina propria. In in vivo conditions solute diffuses through the capillary wall into the blood stream and is carried away. In in vitro conditions it either diffuses through the muscle layer or some may pass out through the capillaries into the fluid bathing the serosal side of the intestine.

From the point of view of compartments and accessibility of these the in vivo condition is complicated. Even if the epithelial cell layer can be regarded as a single membrane, there are at least three compartments, (1) the lumen of the intestine, (2) the fluid in the subepithelial space, (3) the blood stream. The luminal fluid is easily accessible while the subepithelial space is not accessible at all. The blood is accessible but with some limitations. If only concentration of substance is required the problem is simple, but if the total amount of substance entering the blood stream is required then the situation is more complex. Either the blood flow must be measured, or the technique of Matthews \& Smyth (I954) can be used in which the mesenteric venous blood draining the loop is collected. In any event accurate measurements of amounts of substance transferred and concentration changes in the different compartments are extremely difficult in vivo.

In vitro the problem is less complicated. The gut wall is bathed on one side by the mucosal fluid and on the other by the serosal fluid. We have got three compartments, the mucosal fluid, the gut wall and the serosal fluid and we can measure concentration or amount of solute in any of these compartments. The position can be further simplified by thinking of two compartments, the mucosal and serosal 
fluid separated by a complex membrane, the intestinal wall. This problem has been discussed by Barry \& Smyth (1960) who have pointed out that the serosal fluid cannot be regarded as a separate compartment but it is only a part of the compartment into which transfer occurs, since the total compartment includes also the subepithelial space. Furthermore it is a variable part of this compartment and in conditions such as starvation, where the size of the gut changes, the capacity of the subepithelial space could undergo a relative change. By more complicated procedures more compartments have been recognized, and McDougall, Little \& Crane ( 1960 ) studied the epithelium, the core (of the villi), the submucosa and the muscularis mucosa. Crane \& Mandelstam (I960) used the 'tissue' compartment, and this appears to mean the wet weight of the tissue $\times 0.8$. Saunders $\&$ Isselbacher (I965) have used the intracellular compartment and have determined the amount and concentration of solute in this compartment. The method involves estimation of the total water in the tissue, estimation of extracellular water, estimation of the total solute in the gut and estimation of the concentration in the surrounding medium. From these the amount of solute in the cell is calculated by subtracting from the total amount in the gut the amount in the extracellular fluid. This is calculated by assuming that the extracellular fluid is in equilibrium with the bathing medium. Furthermore the calculation depends on assuming that the substance used for determination of extracellular space behaves in exactly the same way as that whose transfer is being studied except that it cannot enter the epithelial cells. These seem doubtful assumptions as the extracellular fluid marker is in equilibrium, or should be, while the substance transferred is at best in a steady state, and it is difficult to see how the extracellular concentrations could be the same as that in surrounding medium, so long as transfer is going on.

\section{Effect of simultaneous fluid transfer}

Simultaneous movement of solvent and solute adds a further complication to the simple two-compartment scheme, to which equation $I$ is applicable. There are four different relationships which are possible between solute and solvent. These are: (I) solvent drag, (2) solute drag, (3) independent solute and solvent movement and (4) the capacity effect of solvent movement.

(I) Solvent drag is the movement of solute caused by movement of solvent. It could occur either in transcellular movement or movement from the subepithelial space into the serosal fluid. The solute affected would depend on the site of solvent drag. If entry from the lumen into the epithelial cell is being considered, then solvent drag could only affect those molecules which can pass through the aqueous pores. On the other hand, in movement from subepithelial space to serosal fluid larger molecules could be involved. There is not much experimental evidence available on the extent to which solvent drag influences solute movement, but Fisher (1955) has produced evidence that it does so in the case of urea, creatine and sorbitol, and the effectiveness of solvent drag decreases with increase in molecular size. Newey \& Smyth (I962) have used procedures for investigating the extent to which solvent drag might play a part in transfer processes. 
(2) By solute drag is meant the movement of solvent occurring as a result of solute movement. This is likely to produce serious errors in assessment of transfer capacity if the parameters used involve concentrations. The transfer of sodium is likely to be most seriously affected, as $\mathrm{Na}$ transfer usually involves a nearly proportional amount of water movement, and hence the solute movement could be completely masked by the accompanying fluid movement.

(3) In some instances there may be some relative independence of solute and solvent. Barry, Smyth \& Wright (1965) have discussed the movement of water due to presence of glucose. One fraction is due to movement of glucose (solute drag of glucose) while another is due to movement of $\mathrm{Na}$ dependent on glucose metabolism (solute drag of $\mathrm{Na}$ ). Clearly the concentration of $\mathrm{Na}$ or of glucose could be affected by the water moving as a result of movement of the other.

(4) The capacity effect of fluid movement is the name given by Barry, Jackson \& Smyth (I966) to another means by which solvent movement might influence solute movement apart from solvent drag. If the epithelial cell layer is capable of moving solute so as to achieve a certain limiting concentration, then the total amount transferred could depend partly on the volume of fluid into which the solute is being moved. Simultaneous movement of fluid would increase this volume, and hence the total amount of solute transferred could be increased before the limiting concentration is reached.

\section{Electrical changes}

When ions are being transferred from one compartment to another equation I can be modified to include a term for the electrical work. There is however a complication which has recently arisen due to the fact that transfer of non-electrolytes may also be associated with an electrical potential. This was shown by Barry, Dikstein, Matthews \& Smyth (I96I) and has been verified by many other workers. The cause of the potential is still unknown. According to Barry et al. (1965) most of the sodium transfer by the intestine is a non-electrogenic process. Schultz \& Zalusky (r964) believe that $\mathrm{Na}$ and glucose move together on one carrier and the $\mathrm{Na}$ is responsible for the hexose-dependent potential. Until this problem is resolved it is not possible to make any calculation of the electrical work related to hexose transfer.

\section{Choice of parameters}

The above discussion shows that it is difficult with existing techniques to assess absorptive ability in terms of work done, and indeed for some purposes it may be irrelevant. Many authors have studied the effect of various conditions on absorption and they have all used either the amounts of substance absorbed or the concentration changes produced by absorption. Accordingly all parameters can be discussed under one or other of these headings.

\section{Amount of substance transferred}

Solute is taken up by the epithelial cells from the intestinal lumen and transferred first to the subepithelial fluid and subsequently, in vivo, to the blood, or in vitro 
to the serosal fluid. What is the best parameter for assessing the amount of solute moved? Consider first the in vivo conditions. The amount leaving the intestinal lumen can be measured in various ways, e.g. various types of intubations, disappearance from tied loops or such procedures as those of Sols \& Ponz (1947) or Sheff \& Smyth (1955). The amount entering the blood stream can be measured, but not very easily, and in vivo the measurement most likely to be made is the amount leaving the intestinal lumen. In vitro both sides of the gut are accessible; the amount of solute leaving the mucosal fluid (mucosal transfer) or the amount entering the serosal fluid (serosal transfer) can be measured. The undesirability of using serosal transfer as a measure of transfer capacity has already been referred to in discussing the compartments involved in absorption. Mucosal transfer is therefore to be preferred for expressing transfer, but there are certain difficulties about this. In the first place if measured by the difference between initial and final amount in the mucosal fluid, it may not be very accurate because it may be a small difference between two larger quantities each with an experimental error. Newey $\&$ Smyth (I962) have preferred to measure the total amount in the gut wall and in the serosal fluid and regard this as the amount transferred. This approach is applicable to all substances not metabolized, but in the case of glucose other considerations must be made. In the first place, because some of the substance is metabolized the amount in the tissue and in the serosal fluid will not give the amount entering the tissue. But even if we determine the amount entering the tissue we do not know whether this represents the amount being actively transferred, as this depends on the spatial relation of metabolic and transfer mechanisms. For a discussion of the problem see Newey \& Smyth (I966). Furthermore in starvation or other abnormal conditions capacity for metabolism might vary as distinct from capacity for transfer. This particular complication does not apply to substances not metabolized, but even in these cases mucosal transfer does not tell us anything about the concentrating capacity of the gut. A further problem is whether to consider the transfer capacity of the gut as the total amount of substance transferred by the whole gut or unit length or weight of gut. In starvation the weight of intestine is reduced and it makes a considerable difference whether we express transfer capacity as the total amount of substance transferred or as the amount transferred by unit weight of intestine. This complication however only arises when the experiment involves changes in gut weight and is not a problem in the large majority of cases.

It is thus evident that while the amount of substance transferred by the gut can be measured, and can be used as an index of transfer capacity, it is by no means the ideal measure of this, and apart from the difficulties discussed here its main weakness is that it fails to give any measure of the concentrating ability.

\section{Concentration changes}

Parameters depending on concentration differences are largely confined to in vitro preparations, and several different parameters have been used. Among these are: (I) the ratio of intracellular concentration to concentration in the surrounding medium, (2) the ratio of the concentration in the gut wall to that in the surrounding 
medium, (3) the ratio between the final serosal concentration and the final mucosal concentration, (4) the difference between the final serosal concentration and the final mucosal concentration, (5) the concentration of substance in the fluid entering or leaving the intestine, (6) increase in serosal concentration.

(1) The ratio of intracellular concentration to concentration in the surrounding medium was used by Saunders \& Isselbacher (1965) and Schultz, Fuisz \& Curran (ı g66). It undoubtedly gives some measure of the capacity of some part of the intestine to concentrate substances from the surrounding medium, but does not necessarily give information about transfer capacity as distinct from concentrating capacity. It must be remembered that cells of many types concentrate substances, but very few are transporting cells in the sense that their main function is to transport substances across the cell. It is therefore possible that non-epithelial elements in the gut wall have a concentrating ability. The technique for measuring intracellular fluid presumably includes all the cells in the gut wall, although authors using this procedure base their arguments on the assumption that they have demonstrated increased concentration in the epithelial cell. Furthermore, it does not necessarily follow that the epithelial transporting cells concentrate within themselves the substances transferred, for this would depend on the cellular location of the transfer process. In the extreme cases if the transfer mechanism is at the luminal pole of the cell there will be increased concentration of transferred substances in the whole cell, while if the transfer process is at the serosal pole of the cell there will be no increased concentration in the cell but indeed there would be a decrease. A third problem already referred to is the difficulty of measuring the amounts of substance in the intracellular compartment.

(2) The ratio of the concentration of substance in the gut wall to that in the surrounding medium has been used by Crane \& Mandelstam (1960), Robinson \& Felber (1965) and Bronk \& Parsons (1966). A ratio greater than unity certainly indicates the ability of the gut to concentrate substances, and wherever this transfer mechanism is located in the epithelial cell there will be an increased concentration in the gut wall as a whole. This ratio could certainly be affected by fluid accompanying solute movement. The ratio will also be affected by the particular experimental conditions, e.g. initial concentration, and a reference to Table 2 in Crane $\&$ Mandelstam (1960) will show the large variations in the ratio which are possible.

(3) The ratio of final serosal to final mucosal concentration has been used by Wiseman (1955). It has the advantage of simplicity and involves a minimum number of measurements with consequent reduction in number of errors. It may be affected by simultaneous movement of fluid with solute, and could not be used as a reliable measure of $\mathrm{Na}$ transfer. Even if the concentrations are initially equal in the mucosal and serosal fluids there could be a period of diffusion into the gut wall from either side, and this could affect the ratio without any active transfer being involved. The final concentration ratio will also depend on the initial amount of solute and fluid volumes present. In the case of substances metabolized the effect of this must be considered in interpreting the significance of the ratio.

(4) The final concentration difference was used by Parsons, Smyth \& Taylor 
(1958) and by others. It has most of the advantages and disadvantages of the final concentration ratio. It might be less affected by the initial volume and amount than the concentration ratio, but this requires experimental investigation.

(5) The concentration of substances in the fluid entering or leaving the gut has been used by Jervis \& Smyth (I960) and by Taylor (I963). It has the disadvantage of relative inaccuracy, particularly when the amount of fluid movement is small. Barry \& Smyth (1960) have pointed out that a high value in the concentration of solute entering the gut can be obtained when no active transfer process is taking place while a high value of the concentration in the fluid entering the serosal compartment is a reliable indication of active transfer.

(6) The change in the serosal concentration has not often been used as a measure of transfer ability although the data are usually available for its calculation. It could be expressed either as a ratio of final serosal to initial serosal concentration or as the difference between these. Provided the initial mucosal concentration of a solute is not greater than the initial serosal concentration, and provided there is no decrease in serosal volume, then an increase in the serosal concentration must indicate active transfer. We are excluding from consideration such complications as ion movement due to electrical potential or such cases as the Brodie-Hogben mechanism which really involves different species. Such complications will of course affect all the parameters.

\section{Conclusions}

The parameters for expressing the ability of the intestine to transfer substances fall into two groups, those expressing the amount transferred and those expressing the concentration changes produced. No one parameter by itself is entirely satisfactory and it would probably be better always to use at least two parameters, one related to the amount of substance transferred and one to the concentration changes produced. The use of different parameters by different authors probably explains some of the apparent contradictions in the literature of intestinal absorption and some of these will be removed when the limitations of different techniques are more fully appreciated.

\section{REFERENCES}

Barry, R. J. C., Dikstein, S., Matthews, J. \& Smyth, D. H. (r96r). F. Physiol., Lond. r55, I7P.

Barry, R. J. C., Jackson, M. J. \& Smyth, D. H. (г966). F. Physiol., Lond. 182, 150.

Barry, R. J. C. \& Smyth, D. H. (1960). F. Physiol., Lond. 152, 48.

Barry, R. J. C., Smyth, D. H. \& Wright, E. M. (1965). F. Physiol., Lond. r8r, 410.

Bronk, J. R. \& Parsons, D. S. (1966). F. Physiol., Lond. 184, 942.

Crane, R. K. \& Mandelstam, P. (1960). Biochim. biophys. Acta 45, 460.

Fisher, R. B. (1955). Y. Physiol., Lond. 130, 655 .

Fisher, R. B. \& Parsons, D. S. (1949). F. Physiol., Lond. r1o, 36.

Jervis, E. L. \& Smyth, D. H. (1960). F. Physiol., Lond. r5x, 5 I.

McDougal, D. B. Jr, Little, K. D. \& Crane, R. K. (I96o). Biochim. biophys. Acta 45, 483.

Matthews, D. M. \& Smyth, D. H. (1954). F. Physiol., Lond. 126, 96.

Newey, H. \& Smyth, D. H. (1962). F. Physiol., Lond. 164, 527.

Newey, H. \& Smyth, D. H. (1966). In D-glucose and Carbohydrates Dependent upon Glucose in Biology and Medicine. [H. Bartelheimer, W. Heyde and W. Thorn, editors.] Berlin: Springer.

Parsons, B. J., Smyth, D. H. \& Taylor, C. B. (1958). F. Physiol., Lond. 144, 387.

Quastel, J. H. (I961). Meth. med. Res. 9, 273. 
Robinson, J. W. L. \& Felber, J. P. (1965). Gastroenterologia, Basel ro4, 335.

Saunders, S. J. \& Isselbacher, K. J. (1965). Biochim. biophys. Acta 102, 397.

Schultz, S. G., Fuisz, R. E. \& Curran, P. F. (1966). F. gen. Physiol. 49, 849.

Schultz, S. G. \& Zalusky, R. (1964). F. gen. Physiol. 47, 1043.

Sheff, M. F. \& Smyth, D. H. (I955). F. Physiol., Lond. 128, 67P.

Smyth, D. H. (196r). Meth. med. Res. 9, 273.

Smyth, D. H. (1963). In Recent Advances in Physiology, p. 36. [R. Creese, editor.] London: J. \& A. Churchill.

Sols, A. \& Ponz, F. (1947). Revta esp. Fisiol. 3, 207.

Taylor, C. B. (1963). F. Physiol., Lond. 165, 199.

Wilson, T. H. (1962). Intestinal Absorption. Philadelphia and London: W. B. Saunders Co.

Wilson, T. H. \& Wiseman, G. (1954). \%. Physiol., Lond. 123, I 16.

Wiseman, G. (1955). F. Physiol., Lond. 127, 4r 4.

\title{
Chairman : Professor SIR HaNs KREBS, Department of Biochemistry, University of Oxford
}

\section{The inhibition and mechanism of intestinal absorption}

\author{
By P. A. SAnford, Department of Physiology, University of Sheffield
}

In studying the mechanisms by which substances presented to the intestine are transferred across the mucosal epithelial cell use has been made of a wide range of inhibitors. These may be classified as ( 1 ) inhibitors which reduce transfer by blocking specific sites or carriers involved in the movement of substances across the mucosal epithelial cell, (2) compounds affecting specific metabolic pathways concerned with providing energy for active transport, (3) inhibitors which, although not directly interfering with the breakdown of metabolizable substrate to provide energy, dissociate this process from active transport. In this paper several inhibitors are considered and their values demonstrated in understanding the mechanisms of intestinal absorption.

\section{Phlorrhizin}

The movement across the small intestine of actively transported sugars, e.g. glucose and galactose, is specificially inhibited by the glycoside phlorrhizin (Fig. I). Many other inhibitors have been found to reduce this movement but none to exhibit so definite an effect at such low concentrations. Since the initial observation of Nakazawa (1922) the attention of many workers has been directed to the problem of how phlorrhizin inhibits sugar transfer. Glucose absorption in the small intestine and the functionally similar kidney was considered to involve phosphorylation and dephosphorylation in the absorbing cells (see Verzár \& McDougall, I936) and, since phlorrhizin was known to inhibit phosphorylation, Lundsgaard (I933) suggested that this effect might explain the observed reduction in glucose movement. Lundsgaard (1935) later rejected this possibility as higher concentrations of phlorrhizin were required to inhibit phosphorylation than were required to inhibit renal tubular glucose reabsorption. Convincing evidence that the reduction of intestinal glucose absorption is not due to inhibition of alkaline phosphatase was provided by Jervis, Johnson, Sheff \& Smyth (1956). Using a phlorrhizin concentration about rooo 